\title{
SOME EXAMPLES OF INVARIABLY GENERATED GROUPS
}

\author{
ASHOT MINASYAN
}

\begin{abstract}
A group $G$ is invariably generated (IG) if there is a subset $S \subseteq G$ such that for every subset $S^{\prime} \subseteq G$, obtained from $S$ by replacing each element with a conjugate, $S^{\prime}$ generates $G$. Likewise, $G$ is finitely invariably generated (FIG) if, in addition, one can choose such a subset $S$ to be finite.

In this note we construct a FIG group $G$ with an index 2 subgroup $N \triangleleft G$ such that $N$ is not IG. This shows that neither property IG nor FIG is stable under passing to subgroups of finite index, answering questions of Wiegold and Kantor-Lubotzky-Shalev. We also produce first examples of finitely generated IG groups that are not FIG, answering a question of Cox.
\end{abstract}

\section{INTRODUCTION}

A subset $S$ invariably generates a group $G$ if for every function $f: S \rightarrow G$ the subset $\left\{s^{f(s)} \mid\right.$ $s \in S\}$ is a generating set of $G$. We say that $G$ is invariably generated (IG) if it contains an invariable generating subset (equivalently, if $G$ invariably generates itself). Similarly, $G$ is finitely invariably generated (FIG) if it has a finite invariable generating subset.

The term "invariably generated" was invented by Dixon [6] in 1988, though the notion itself appeared in the literature earlier. In 1975 Wiegold [26] considered the class of groups $\mathcal{X}$ such that $G \in \mathcal{X}$ if and only if for every transitive action of $G$ on a set $\Omega$, with $|\Omega| \geq 2$, at least one element $g \in G$ acts on $\Omega$ without fixed points. The fact that the latter property holds for all finite groups was proved by Jordan [12] in 1872, more that 100 years earlier. Jordan's theorem was revisited by Serre [24] in 2003, who gave several applications to Number Theory and Topology.

In [26] Wiegold observed the following.

Remark 1.1. For a group $G$ the following statements are equivalent:

- $G \in \mathcal{X}$

- for each proper subgroup $H<G, \bigcup_{g \in G} H^{g} \neq G$;

- if $S$ is a subset of $G$ containing a representative from each conjugacy class, then $G=\langle S\rangle$; in other words, $G$ is IG.

Given two subsets $A, B$ of a group $G$ we will say that $A$ is pointwise conjugate into $B$ if $A \subseteq B^{G}$, i.e., each $a \in A$ is conjugate to some $b \in B$. The above remark tells us that $G$ is IG if and only if $G$ is not pointwise conjugate into a proper subgroup. Wiegold proved that the class $\mathcal{X}$ of IG groups is closed under extensions and restricted direct products [26]. Since finite groups (by Jordan's theorem [12]) and abelian groups are in $\mathcal{X}$, it follows that all virtually solvable groups are IG. On the other hand, the easiest examples of non-IG groups are non-abelian free groups [26].

In 2014 Kantor, Lubotzky and Shalev [13] studied invariable generation for infinite linear groups and defined the property FIG. Similarly to Remark 1.1, a group is FIG if and only if there is a finite subset $S \subseteq G$ which is not pointwise conjugate into any proper subgroup of $G$. One of the main results from [13] states that a finitely generated linear group is FIG if and only if it is virtually solvable. They also showed that the class of FIG groups is closed under extensions and contains all finitely generated abelian-by-polycyclic groups. In fact, the argument from [13, Lemma 2.8] (see also [26, Theorem 2.1.(i)]) proves the following.

Lemma 1.2. Suppose that $G$ is a group, $N \leqslant K \leqslant G$ and $N \triangleleft G$. If $S$ is an invariable generating subset of $K$ and $T$ is an invariable generating subset of $G / N$ then $S \cup T_{1}$ is an invariable generating subset of $G$, where $T_{1} \subseteq G$ is any preimage of $T$ in $G$.

2010 Mathematics Subject Classification. 20F67, 20F65, $20 \mathrm{~F} 06$.

Key words and phrases. Invariably generated, finitely invariably generated, finite index subgroups. 
This lemma easily yields that each of the properties IG and FIG is inherited by finite index overgroups. Wiegold [27, p. 573] and Kantor, Lubotzky, Shalev [13, Open Problems 1,2] asked whether the same can be said for finite index subgroups. Our first result gives a negative answer to these two questions.

Theorem 1.3. There exists a torsion-free group $G$ with a subgroup $N \triangleleft G$, of index 2 , such that $G$ is invariably generated by two elements but $N$ is not invariably generated. More precisely, there are two elements $a, b \in G$ such that all of the following hold.

(i) $\{a, b\}$ invariably generates $G$;

(ii) the subgroup $N=\left\langle a^{2}, b, a b a^{-1}\right\rangle$ has index 2 in $G$;

(iii) $H=\left\langle a^{2}, b\right\rangle$ is free of rank 2 , freely generated by $\left\{a^{2}, b\right\}$, and $|N: H|=\infty$;

(iv) $N=\bigcup_{f \in N} H^{f}$.

Note that the first example of a non-IG subgroup of an IG group was given by Wiegold in [27].

More examples of IG, FIG and non-IG infinite groups have been recently obtained in $[7,8,3$, 5]. Property FIG implies IG, by definition; in fact, FIG is strictly stronger as any non-finitely generated abelian group is IG but not FIG. However, prior to this work none of the known examples answered the following basic question, asked by Cox in [5]: is property IG equivalent to FIG for finitely generated groups? Our second theorem shows that this is not the case.

Theorem 1.4. There exists a finitely generated torsion-free group $G$ which is IG but not FIG. More precisely, $G$ is generated by two elements and there is an infinite family of subgroups $H_{j}<G$, $j=0,1,2, \ldots$, such that all of the following conditions are satisfied.

(i) For all $j \in \mathbb{N} \cup\{0\}, H_{j}$ is a malnormal finitely generated free subgroup of $G$;

(ii) for each $i \in \mathbb{N}$ there exists $r \in H_{i} \backslash\{1\}$ such that $\langle r\rangle \cap g H_{j} g^{-1}=\{1\}$, for any $j<i$ and all $g \in G$;

(iii) every finite subset of $G$ is pointwise conjugate into $H_{j}$, for some $j \in \mathbb{N} \cup\{0\}$;

(iv) the intersection $h H_{j} h^{-1} \cap H_{i}$ is cyclic, for all $i \neq j$ and all $h \in G$;

(v) if $M$ is a proper subgroup of $G$, then there exists $j \in \mathbb{N} \cup\{0\}$ and $v \in G$ such that $M \subseteq v H_{j} v^{-1}$.

Recall that a subgroup $H$ of a group $G$ is called malnormal if $g H g^{-1} \cap H=\{1\}$ for all $g \in G \backslash H$. The fact that any group $G$ satisfying properties (i)-(v) from Theorem 1.4 is not FIG follows from (iii). Condition (ii) shows that $G \neq \bigcup_{g \in G} H_{j}^{g}$ for any given $j \in \mathbb{N} \cup\{0\}$, and since each proper subgroup of $G$ is conjugate into some $H_{j}$, by (v), we can use Remark 1.1 to conclude that $G$ is IG.

Thus even for finitely generated groups property FIG is more restrictive than property IG. In view of Theorems 1.3 and 1.4, [5, Corollary A] implies that every finitely generated group can be embedded in a FIG group that has a non-IG subgroup of finite index or in a finitely generated IG non-FIG group. In particular, there is a continuum of such groups.

After this paper was written, the author learned that Goffer and Lazarovich obtained similar results to Theorems 1.3,1.4 independently in [9]. Their work also answers the questions of Wiegold, Kantor-Lubotzky-Shalev and Cox mentioned above. Both our paper and [9] are based on the small cancellation theory over hyperbolic groups developed by Ol'shanskii [21]. However, [9] works with this theory directly, while our approach uses the author's previous results from [18].

1.1. Ideas of proofs. Let us briefly outline the constructions of the groups from Theorems 1.3 and 1.4. In both of them the group $G$ is obtained as a direct limit of torsion-free hyperbolic groups, using a "small cancellation quotient theorem". In the original form such a theorem was proposed by Gromov [10, 5.5], and then proved by Ol'shanskii in [21]. The statement has later been extended to relatively hyperbolic groups by Osin [23] and then to acylindrically hyperbolic 
groups by Hull [11]. The version that we will use here was obtained by the author in [18, 17] and allows to preserve certain quasiconvex subgroups in the small cancellation quotient: see Theorem 2.6 below. This version has an advantage over the versions from $[23,11]$ in not having to require that the preserved collection of quasiconvex subgroups be malnormal in the ambient hyperbolic group, which is important for the constructions that we present here.

To prove Theorem 1.3 we start with a group $G_{0}$, freely generated by $\left\{a_{0}, b_{0}\right\}$, let $H_{0}=\left\langle a_{0}^{2}, b_{0}\right\rangle$ and $N_{0}=\left\langle a_{0}^{2}, b_{0}, b_{0}^{a_{0}}\right\rangle \triangleleft G_{0}$. We enumerate all elements of $G_{0}: f_{0}=1, f_{1}, f_{2}, \ldots$, and construct non-elementary torsion-free hyperbolic groups $G_{1}, G_{2}, \ldots$ by induction, so that for each $G_{n+1}$ is a quotient of $G_{n}, n \geq 0$. To obtain $G_{n+1}$ from $G_{n}$, together with an epimorphism $\phi_{n+1}: G_{n} \rightarrow$ $G_{n+1}$, we first embed $G_{n}$ into an HNN-extension $L_{n}$, in which the image of $f_{n+1}$ is conjugate to an element of the image of $H_{0} \cup\left\langle a_{0}\right\rangle$. We then use Theorem 2.6 to produce a torsion-free hyperbolic group $G_{n+1}$, and an epimorphism $\eta_{n+1}: L_{n} \rightarrow G_{n+1}$ such that $\eta_{n+1}$ is injective on the image of $H_{0}$ in $L_{n}$ and $G_{n+1}$ is generated by the images of $a_{0}$ and $b_{0}^{f_{n+1}}$. In particular, $\eta_{n+1}\left(G_{n}\right)=G_{n+1}$ and we set $\phi_{n+1}: G_{n} \rightarrow G_{n+1}$ to be the restriction of $\eta_{n+1}$ to $G_{n}$. We define the epimorphism $\psi_{n+1}: G_{0} \rightarrow G_{n+1}$ by $\psi_{n+1}=\phi_{n+1} \circ \cdots \circ \phi_{1}$, and set $a_{n+1}=\psi_{n+1}\left(a_{0}\right), b_{n+1}=\psi_{n+1}\left(b_{0}\right)$, $H_{n+1}=\psi_{n+1}\left(H_{0}\right)$ and $N_{n+1}=\psi_{n+1}\left(N_{0}\right)$. By carefully controlling the kernel of $\eta_{n+1}$ we ensure that $N_{n+1}$ still has index 2 in $G_{n+1}$ and $\psi_{n+1}\left(f_{n+1}\right)$ is conjugate into $H_{n+1} \cup\left\langle a_{n+1}\right\rangle$ via an element of $N_{n+1}$ in $G_{n+1}$. Finally we define $G$ as the direct limit of the sequence $\left(G_{n}\right)_{n=0}^{\infty}$, let $a, b \in G$ and $H, N \leqslant G$ be the natural images of $a_{0}, b_{0} \in G_{0}$ and $H_{0}, N_{0} \leqslant G_{0}$ respectively. By construction, every $G_{i}$ is generated by $a_{i}$ and $b_{i}^{\psi_{i}\left(f_{i}\right)}$, whence $G=\left\langle a, b^{f}\right\rangle$ for all $f \in G$, so that $\{a, b\}$ is an invariable generating set of $G$. On the other hand, the condition that $\psi_{i}\left(f_{i}\right) \in H_{i}^{N_{i}} \cup\left\langle a_{i}\right\rangle^{N_{i}}$ in $G_{i}$, $i \in \mathbb{N}$, is sufficient for showing that $N=\bigcup_{f \in N} H^{f}$. The actual argument is somewhat technical, since various conditions need to be preserved at each step in order for the inductive argument to work and for Theorem 2.6 to be applicable.

The proof of Theorem 1.4 is more involved because the difference between properties IG and FIG is quite subtle. We start with a free group $G_{0}$ of rank 3 , set $H_{00}=\{1\}$, and enumerate all of the finite subsets $S_{0}=\emptyset, S_{1}, S_{2}, \ldots$, and all of the finitely generated subgroups $Y_{0}=\{1\}$, $Y_{1}, Y_{2}, \ldots$ in $G_{0}$. The torsion-free hyperbolic group $G_{n+1}$, together with a collection of malnormal quasiconvex subgroups $H_{n+1, j} \leqslant G_{n+1}, j=0, \ldots, n+1$, are constructed by induction on $n$. We define $L_{n}$ as an HNN-extension of the free product $G_{n} * X$, where $X$ is a free group of finite rank and the image of $S_{n+1}$ is pointwise conjugate into $X$ in $L_{n}$. Thereafter $G_{n+1}$ is produced as a small cancellation quotient of $L_{n}$ in such a way that the natural homomorphism $\eta_{n+1}: L_{n} \rightarrow G_{n+1}$ is surjective on $G_{n}$ and on the image of $Y_{n+1}$ in $G_{n}$, unless this image is cyclic or is contained in a conjugate of $H_{n j}$ in $L_{n}$, for some $j=0, \ldots, n$. We let $H_{n+1, j} \leqslant G_{n+1}$ be the image of $H_{n j}$, for $j \leq n$, and $H_{n+1, n+1}=\eta_{n+1}(X)$. As before, $G$ is defined as the direct limit of the sequence $\left(G_{n}\right)_{n=0}^{\infty}$, and for each $j \geq 0, H_{j}$ is defined as the image of $H_{j j}$ under the natural epimorphism $G_{j} \rightarrow G$. It is straightforward from the construction that every proper finitely generated subgroup of $G$ (being the image of some $Y_{j}$ ), will be contained in a conjugate of $H_{j}$, for some $j \geq 0$. The much stronger condition (v) is proved by combining the malnormality of each subgroup $H_{j j} \leqslant G_{j}$ with property (iv) from the claim of the theorem.

The constructions we use to prove Theorems 1.3,1.4 are fairly flexible and one can produce examples with additional properties: see Remarks 3.1,3.2 and 4.5.

Acknowledgements. The author would like to thank Charles Cox whose talk on the paper [5] motivated this work, and who gave useful comments on a preliminary version of this article. The author also thanks the anonymous referee for many useful suggestions which led to improvements of the exposition. 


\section{Preliminaries}

2.1. Notation and terminology. In this paper we will denote by $\mathbb{Z}$ the set of all integers, by $\mathbb{N}=\{1,2, \ldots\}$ the set of all natural numbers, and by $2 \mathbb{N}=\{2,4, \ldots\}$ the set of all even natural numbers.

Let $G$ be a group, $x, y \in G$ be any elements and $A, B \subseteq G$ be any subsets. We will use the following notation:

$$
x^{y}=y x y^{-1}, A^{x}=x A x^{-1}, A^{B}=\left\{b a b^{-1} \mid a \in A, b \in B\right\} .
$$

We will say that $x$ is commensurable to $y$ in $G, x \underset{G}{\approx} y$, if some non-zero power of $x$ is conjugate to some non-zero power of $y$ in $G$. Note that $\underset{\approx}{\approx}$ is an equivalence relation on $G$.

As usual, $\mathrm{C}_{G}(x)=\{g \in G \mid g x=x g\}$ will denote the centralizer of $x$ in $G$.

Definition 2.1. Let $\eta: L \rightarrow G$ be a homomorphism between groups $L$ and $G$, and let $Q \subseteq L$ be any subset. We will say that $\eta$ preserves conjugacy on $Q$ if for all $x, y \in Q, \eta(y) \in \eta(x)^{\bar{G}}$ in $G$ implies that $y \in x^{L}$ in $L$. We will also say that $\eta$ preserves centralizers on $Q$ if $\mathrm{C}_{G}(\eta(x))=$ $\eta\left(\mathrm{C}_{L}(x)\right)$ for all $x \in Q$.

Finally, recall that a group is called elementary if it has a cyclic subgroup of finite index. Throughout the paper we will often use the basic fact that a torsion-free elementary group is necessarily cyclic.

2.2. One subgroup of a free group. The following elementary facts about a specific subgroup of a free group of rank 2 will be used in the proof of Theorem 1.3.

Lemma 2.2. Let $G$ be a free group of rank 2 with free generating set $\{a, b\}$, let $H=\left\langle a^{2}, b\right\rangle \leqslant G$ and $N=\left\langle a^{2}, b, b^{a}\right\rangle \leqslant G$. Then

(i) $N \triangleleft G$ and $|G: N|=2$, in particular, $|N: H|=\infty$;

(ii) $H \cap H^{a}=\left\langle a^{2}\right\rangle$;

(iii) if $a^{l} \in h^{G}$, for some $h \in H$ and some $l \in \mathbb{Z}$, then $a^{l} \in h^{H}$;

(iv) $\mathrm{C}_{G}\left(a^{l}\right)=\langle a\rangle$ for any $l \in \mathbb{Z} \backslash\{0\}$, and $\mathrm{C}_{G}(u) \subseteq H$ if $u \in H \backslash\left\langle a^{2}\right\rangle^{H}$.

Proof. Claim (i) follows from the observation that $N$ is the kernel of the homomorphism from $G$ onto $\mathbb{Z} / 2 \mathbb{Z}$, sending $a$ to $\overline{1}$ and $b$ to $\overline{0}$, which also implies that $\left\{a^{2}, b, b^{a}\right\}$ is a free generating set of $N$. Therefore $H \cap H^{a}=\left\langle a^{2}\right\rangle$, as $H^{a}=\left\langle a^{2}, b^{a}\right\rangle$, so (ii) holds.

Now, suppose that $h=x a^{l} x^{-1} \in H$ for some $x \in G$ and $l \in \mathbb{Z}$. Since $a \notin N$ and $H \subset N$ we see that $l$ must be even, so $a^{l} \in H$. As $|G: N|=2$, there exist $k \in\{0,1\}$ and $t \in N$ such that $x=t a^{k}$, so $h=t a^{l} t^{-1}$ in $N$. Observe that $H$ is malnormal in $N$, being a free factor, hence either $l=0$ and $h=1$ or $t \in H$. This proves claim (iii).

The fact that $\mathrm{C}_{G}\left(a^{l}\right)=\langle a\rangle$, for any $l \in \mathbb{Z} \backslash\{0\}$, is obvious. Now suppose that $u \in H$ is an element such that $\mathrm{C}_{G}(u) \nsubseteq \nsubseteq H$. Since $H$ is malnormal in $N$, there must exist $v \in \mathrm{C}_{G}(u) \backslash N$. Thus $v=f a$ for some $f \in N$, so $u=u^{v}=f u^{a} f^{-1}$, and $w=f^{-1} u f=u^{a} \in H^{a}$ is conjugate to $u \in H$ in $N$.

Recall that $N$ is freely generated by $S=\left\{a^{2}, b, b^{a}\right\}$, and $H=\left\langle a^{2}, b\right\rangle, H^{a}=\left\langle a^{2}, b^{a}\right\rangle$ are freely generated by subsets of $S$. Therefore we can write $u=h u_{1} h^{-1}$ and $w=h^{\prime} w_{1} h^{\prime-1}$, where $u_{1}, h \in H, w_{1}, h^{\prime} \in H^{a}$, and the elements $u_{1}, w_{1}$ are cyclically reduced over $S$. The conjugacy criterion for cyclically reduced elements in the free group $N$ (see [15, Proposition I.2.14]) implies that $u_{1}=a^{n}=w_{1}$, for some $n \in 2 \mathbb{N}$. Hence $u=h a^{n} h^{-1} \in\left\langle a^{2}\right\rangle^{H}$, and (iv) holds.

2.3. Hyperbolic groups and quasiconvex subsets. Let $G$ be a hyperbolic group in the sense of Gromov [10]. This means that $G$ is generated by a finite set $S$, and the Cayley graph $\Gamma(G, S)$ is $\delta$-hyperbolic, for some $\delta \geq 0$ (see [4, Part III.H] for a detailed exposition). A subset $Q$ of $G$ is 
said to be quasiconvex if there exists $\varepsilon \geq 0$ such that any geodesic joining two elements of $Q$ in $\Gamma(G, S)$ is contained in the $\varepsilon$-neighborhood of $Q$.

We outline basic properties of quasiconvex subsets in the following remark.

Remark 2.3. Let $G$ be a hyperbolic group and let $Q \subseteq G$. Then

(1) the quasiconvexity of $Q$ is independent of the choice of a finite generating set $S$ for $G$;

(2) any elementary subgroup of $G$ is quasiconvex;

(3) if $G$ is a free group of finite rank then any finitely generated subgroup of $G$ is quasiconvex;

(4) finite unions and products of quasiconvex subsets of $G$ are also quasiconvex;

(5) if $K$ is a quasiconvex subgroup of $G$ then $K$ is itself hyperbolic and any quasiconvex subset of $K$ is also quasiconvex in $G$.

Properties (1)-(3) are well-known (cf. [1, Chapter 3] and [25]); see [16, Lemma 2.1 and Proposition 0.1] for property (4). Finally, property (5) follows from [1, 3.3.8] and [18, Remark 6].

Lemma 2.4. If $G$ is a torsion-free hyperbolic group and $c \in G$ is a non-trivial element then $\mathrm{C}_{G}(c)$ is a malnormal infinite cyclic subgroup of $G$, generated by some $d \in G$. In particular, $\mathrm{C}_{G}\left(d^{m}\right)=\langle d\rangle$, for any $m \in \mathbb{Z} \backslash\{0\}$.

Proof. This is an immediate consequence of [21, Lemma 1.16] and the fact that torsion-free elementary groups are cyclic.

Lemma 2.5. Let $G$ be a torsion-free hyperbolic group and let $x, y, z \in G$ be elements satisfying $y^{l}=z x^{k} z^{-1}$, for some $k, l \in \mathbb{Z} \backslash\{0\}$. Then $y \in \mathrm{C}_{G}(x)^{z}$.

Proof. By Lemma 2.4, $\mathrm{C}_{G}(x)^{z}=\mathrm{C}_{G}\left(x^{z}\right)$ is malnormal in $G$, so, since $G$ is torsion-free, $y^{l} \in \mathrm{C}_{G}(x)^{z}$ implies that $y \in \mathrm{C}_{G}(x)^{z}$.

Following [18] we say that a subset $Q$ of a group $G$ is small relative to a subgroup $F \leqslant G$ if $F \nsubseteq P_{1} Q^{-1} Q P_{2}$ for any finite subsets $P_{1}, P_{2}$ of $G$.

The next result was obtained by the author in [18], as part of his PhD thesis [17], and generalized an earlier theorem of Ol'shanskii [21].

Theorem 2.6. Suppose that $L$ is a torsion-free hyperbolic group, $F \leqslant L$ is a non-elementary subgroup, $Q \subseteq L$ is a quasiconvex subset and $U=\left\{u_{1}, \ldots, u_{n}\right\}$ is any finite collection of elements of $L$. If $Q$ is small relative to $F$ in $L$ then there exist elements $w_{1}, \ldots, w_{n} \in F$ satisfying the following. Let $K \triangleleft L$ be the normal closure of the elements $u_{i} w_{i}, i=1, \ldots, n$, let $G=L / K$ and let $\eta: L \rightarrow G$ be the natural homomorphism. Then

(a) $G$ is a non-elementary torsion-free hyperbolic group;

(b) the restriction of $\eta$ to $Q$ is injective and $\eta(R)$ is quasiconvex in $G$ for every quasiconvex subset $R$ of $L$ with $R \subseteq Q$;

(c) $\eta$ preserves centralizers on $Q$ (in the sense of Definition 2.1);

(d) $\eta$ preserves conjugacy on $Q$ (in the sense of Definition 2.1).

Proof. The statement is essentially a special case of [18, Theorem 1] (indeed, any non-elementary subgroup of a torsion-free hyperbolic group is a $G$-subgroup by [21, Theorem 1]). The main difference is that here we do not require the homomorphism $\phi$ to be surjective on $F$, but we simply ask for the image of $F$ to contain the images of the given elements $u_{i}, i=1, \ldots, n$. This allows us to specify that $\operatorname{ker} \phi=K$ is the normal closure of elements of the form $u_{i} w_{i}$, for some $w_{i} \in F, i=1, \ldots, n$. The latter can be seen from the explicit form of the extra relators imposed on $L$ to obtain $G$ in the proof of [18, Theorem 1]: see equation (21) and Section 7 in [18].

In the case when $Q$ is a finite union of cosets of quasiconvex subgroups the condition of being small relative to $F$ was characterized in [18] as follows. 
Theorem 2.7 ([18, Theorem 3]). Let $L$ be a hyperbolic group and let $F \leqslant L$ be any subgroup. Suppose that $H_{1}, \ldots, H_{k}$ are quasiconvex subgroups of $L$ such that $\left|F:\left(F \cap g H_{i} g^{-1}\right)\right|=\infty$ for all $g \in L$ and all $i=1, \ldots, k$. Then the quasiconvex subset $Q=\bigcup_{i=1}^{k} H_{i} \subseteq L$ is small relative to $F$ in $L$.

The next lemma shows that the map $\eta$ from Theorem 2.6 preserves malnormality of any subgroup contained in $Q$.

Lemma 2.8. Suppose that $\eta: L \rightarrow G$ is a homomorphism between groups $L$ and $G$, and $H$ is a malnormal subgroup of $L$. If $\eta$ preserves conjugacy and centralizers on $H$ then $\eta(H)$ is malnormal in $G$.

Proof. Assume that $d=c^{g}$, for some $c, d \in \eta(H) \backslash\{1\}$ and some $g \in G$. Choose $a, b \in H \backslash\{1\}$ such that $\eta(a)=c$ and $\eta(b)=d$. Since $\eta$ preserves conjugacy on $H, b=a^{h}$, for some $h \in L$, which implies that $h \in H$ as $H$ is malnormal in $L$.

Now, $c^{g}=d=c^{\eta(h)}$ in $G$, so $\eta(h)^{-1} g \in \mathrm{C}_{G}(c)=\eta\left(\mathrm{C}_{L}(a)\right)$, since $\eta$ preserves centralizers on $H$. Moreover, $\mathrm{C}_{L}(a) \subseteq H$, as $H$ is malnormal in $L$, so $g \in \eta(h) \eta\left(\mathrm{C}_{L}(a)\right) \subseteq \eta(H)$. Therefore $\eta(H)$ is malnormal in $G$.

The following statement is essentially a corollary of one of the author's results from [19].

Lemma 2.9. Let $H_{1}, H_{2}$ be subgroups of a hyperbolic group $L$, with $H_{1}$ quasiconvex, let $G$ be any group and let $\eta: L \rightarrow G$ be a homomorphism. Suppose that the following two conditions are satisfied:

(i) $\eta$ preserves conjugacy on $H_{1} \cup H_{2}$;

(ii) the intersection $H_{1}^{g} \cap H_{2}$ is elementary, for every $g \in L$.

Then for all $h \in G$, the intersection $\eta\left(H_{1}\right)^{h} \cap \eta\left(H_{2}\right)$ is elementary in $G$.

Proof. Choose any $h \in G$ and denote $A=\eta\left(H_{1}\right)^{h} \cap \eta\left(H_{2}\right) \leqslant G$. Thus $A \leqslant \eta\left(H_{2}\right)$ is conjugate into $\eta\left(H_{1}\right)$ in $G$. Let $B \leqslant H_{2}$ be a preimage of $A$ under $\eta$. Then $B$ is pointwise conjugate into $H_{1}$ by (i), and, as $L$ is hyperbolic and $H_{1}$ is quasiconvex, we can apply [19, Proposition 1] to conclude that $\left|B:\left(B \cap H_{1}^{g}\right)\right|<\infty$ for some $g \in L$. But $B \cap H_{1}^{g} \subseteq H_{2} \cap H_{1}^{g}$ is elementary by condition (ii), hence $B$ is elementary, and so is $A=\eta(B)$.

2.4. HNN-extensions. We will need the following statements about HNN-extensions.

Lemma 2.10. Let $G$ be a hyperbolic group, let $X=\langle x\rangle, Y=\langle y\rangle$ be infinite cyclic subgroups of G. Suppose that either $X$ or $Y$ is malnormal in $G$ and $x \not \approx y$. Then the HNN-extension

$$
L=G *_{X^{t}=Y}=\left\langle G, t \| t x t^{-1}=y\right\rangle
$$

is hyperbolic, and for any quasiconvex subgroup $H \leqslant G, H$ is quasiconvex in $L$.

Proof. The hyperbolicity of $L$ under the above assumptions was first proved by Bestvina and Feighn [2, Corollary 2.3]. Since cyclic subgroups in hyperbolic groups are always quasiconvex (see Remark 2.3), $G$ will be quasiconvex in $L$ by a result of Kharlampovich and Myasnikov [14, Theorem 4]. Claim (5) of Remark 2.3 now shows that $H$ is quasiconvex in $L$.

Lemma 2.11. Suppose that $G$ is a group and $X, Y \leqslant G$ are isomorphic subgroups, with an isomorphism $\tau: X \rightarrow Y$, and $C \leqslant G$ is any subgroup. Let $L$ be the $H N N$-extension

$$
L=G *_{X^{t}=Y}=\left\langle G, t \| t x t^{-1}=\tau(x), x \in X\right\rangle .
$$

(a) If $C$ and $Y$ are malnormal in $G$ and $C \cap Y^{G}=\{1\}$ then $C$ is malnormal in $L$.

(b) If $u, v \in G$ are elements such that $v \notin u^{G}$ and $u \notin X^{G} \cup Y^{G}$ then $v \notin u^{L}$ in $L$.

Proof. See [18, Lemma 10.1] for (a) and [20, Lemma 3.4] for (b). 


\section{INVARIABLE GENERATION MAY NOT PASS TO FINITE INDEX SUBGROUPS}

Proof of Theorem 1.3. The desired group $G$ will be constructed as a direct limit of torsion-free hyperbolic groups. Let $G_{0}$ be a free group freely generated by two elements $a_{0}, b_{0} \in G_{0}$. Let $N_{0}=\left\langle a_{0}^{2}, b_{0}, a_{0} b_{0} a_{0}^{-1}\right\rangle$, so that $\left|G_{0}: N_{0}\right|=2$, and let $H_{0}=\left\langle a_{0}^{2}, b_{0}\right\rangle$ and $Q_{0}=H_{0} \cup H_{0}^{a_{0}}$. Then $\left\{a_{0}^{2}, b_{0}\right\}$ is a free generating set for $H_{0}$, and $H_{0}, Q_{0}$ are quasiconvex in $G_{0}$ by part (3) of Remark 2.3. Let $\left\{f_{0}=1, f_{1}, f_{2}, \ldots,\right\}$ be an enumeration of all elements of $G_{0}$.

For each $i \in \mathbb{N}$ we will construct a group $G_{i}$ and an epimorphism $\phi_{i}: G_{i-1} \rightarrow G_{i}$, so that for $\psi_{i}=\phi_{i} \circ \cdots \circ \phi_{1}: G_{0} \rightarrow G_{i}, a_{i}=\psi_{i}\left(a_{0}\right) \in G_{i}, b_{i}=\psi_{i}\left(b_{0}\right) \in G_{i}, g_{i}=\psi_{i}\left(f_{i}\right) \in G_{i}$, $N_{i}=\psi_{i}\left(N_{0}\right)=\left\langle a_{i}^{2}, b_{i}, a_{i} b_{i} a_{i}^{-1}\right\rangle \triangleleft G_{i}, H_{i}=\psi_{i}\left(H_{0}\right)=\left\langle a_{i}^{2}, b_{i}\right\rangle \leqslant G_{i}$ and $Q_{i}=\psi_{i}\left(Q_{0}\right)=H_{i} \cup H_{i}^{a_{i}}$ the following conditions hold:

(3.1) $G_{i}$ is a torsion-free hyperbolic group generated by $a_{i}, b_{i}$;

(3.3) $\psi_{i}$ is injective on $Q_{0}, H_{i}$ and $Q_{i}$ are quasiconvex in $G_{i}$;

(3.4) $\mathrm{C}_{G_{i}}\left(a_{i}^{l}\right)=\left\langle a_{i}\right\rangle$, for all $l \in \mathbb{Z} \backslash\{0\}$, and $\mathrm{C}_{G_{i}}(u) \subseteq H_{i}$ if $u \in H_{i} \backslash\left\langle a_{i}^{2}\right\rangle^{H_{i}}$;

(3.5) if $a_{i}^{l} \in h^{G_{i}}$, for some $l \in 2 \mathbb{N}$ and $h \in H_{i}$, then $a_{i}^{l} \in h^{H_{i}}$;

(3.6) $g_{i} \in H_{i}^{N_{i}} \cup\left\langle a_{i}\right\rangle^{N_{i}}$;

(3.7) $G_{i}=\left\langle a_{i}, b_{i}^{g_{i}}\right\rangle$.

Setting $\psi_{0}: G_{0} \rightarrow G_{0}$ to be the identity map, we see that for $i=0$ conditions (3.1)-(3.7) are all satisfied (either by construction or by Lemma 2.2). We now proceed by induction, and thus assume that for some $n \in \mathbb{N} \cup\{0\}$, groups $G_{0}, \ldots, G_{n}$, enjoying properties (3.1)-(3.7), have already been constructed. We are going to construct a group $G_{n+1}$ and an epimorphism $\phi_{n+1}: G_{n} \rightarrow G_{n+1}$ so that (3.1)-(3.7) hold for $i=n+1$.

Denote $c=\psi_{n}\left(f_{n+1}\right) \in G_{n}$. We shall first define an intermediate torsion-free hyperbolic group $L_{n}$, containing $G_{n}$, and an index two normal subgroup $M_{n} \triangleleft L_{n}$ such that $M_{n} \cap G_{n}=N_{n}$ and $c \in\left(H_{n} \cup\left\langle a_{n}\right\rangle\right)^{t}$, for some $t \in M_{n}$. If $c=1$ then set $L_{n}=G_{n}, M_{n}=N_{n}$ and $t=1$. So, assume that $c \neq 1$ and let $d \in G_{n}$ be an infinite order element such that $\mathrm{C}_{G_{n}}(c)=\langle d\rangle$ (cf. Lemma 2.4).

If $d \in H_{n}^{N_{n}}$ in $G_{n}$ then set $L_{n}=G_{n}, M_{n}=N_{n}$ and let $t \in M_{n}$ be any element such that $d, c \in H_{n}^{t}$. Similarly, if $d \stackrel{G_{n}}{\approx} a_{n}$, then $d, c \in \mathrm{C}_{G_{n}}\left(a_{n}\right)^{z}$, for some $z \in G_{n}$, by Lemma 2.5. So, recalling (3.4), we get $d, c \in\left\langle a_{n}\right\rangle^{t}$ in $G_{n}$, where $t \in N_{n}$ is the element such that $z=t a_{n}^{\varepsilon}$, for some $\varepsilon \in\{0,1\}$. Thus we can again take $L_{n}=G_{n}, M_{n}=N_{n}$ and $t$ as above.

So, further we can assume that $d$ is not commensurable to $a_{n}$ in $G_{n}$ and $d \notin H_{n}^{N_{n}}$. Case 1: $d \notin N_{n}$. We claim that $d$ cannot be commensurable to any element from $H_{n}$, so

$$
\langle d\rangle^{G_{n}} \cap H_{n}=\{1\} .
$$

Indeed, if $d \stackrel{G_{n}}{\approx} u \in H_{n}$, then $d$ would be conjugate into $\mathrm{C}_{G_{n}}(u)$ by Lemma 2.5. But $u \notin\left\langle a_{n}\right\rangle^{G_{n}}$, as $d \stackrel{G_{n}}{\not} a_{n}$, hence $\mathrm{C}_{G_{n}}(u) \subseteq H_{n} \subset N_{n}$ by (3.4). The latter would imply that $d \in N_{n}$, because $N_{n}$ is normal in $G_{n}$, contradicting the assumption of this case.

So, in Case 1 we define $L_{n}$ as the HNN-extension

$$
L_{n}=\left\langle G_{n}, t \| t a_{n} t^{-1}=d\right\rangle .
$$

Case 2: $d \in N_{n}$. Since $H_{n} \cong H_{0}$ is non-elementary, by [21, Lemma 3.8] there exists an infinite order element $e \in H_{n}$ such that

$$
e \stackrel{G_{n}}{\not \approx} a_{n}, e \stackrel{G_{n}}{\not \approx} d \text { and } \mathrm{C}_{G_{n}}(e)=\langle e\rangle
$$


Thus in Case 2 we define $L_{n}$ as the HNN-extension

$$
L_{n}=\left\langle G_{n}, t \| t e t^{-1}=d\right\rangle .
$$

Now suppose that we are in one of the above two cases. The group $L_{n}$ is torsion-free and hyperbolic, and $H_{n}$ is quasiconvex in $L_{n}$ by Lemmas 2.4, 2.10. Therefore $Q_{n}=H_{n} \cup H_{n}^{a_{n}}$ is quasiconvex in $L_{n}$ by Remark 2.3. Let $\xi: G_{n} \rightarrow \mathbb{Z} / 2 \mathbb{Z}$ denote the epimorphism with kernel $N_{n}$. Observe that in each of the presentations (3.9), (3.11) above the free letter $t$ conjugates elements which have the same image under $\xi$ in $\mathbb{Z} / 2 \mathbb{Z}$. Therefore $\xi$ can be extended to an epimorphism $\widehat{\xi}: L_{n} \rightarrow \mathbb{Z} / 2 \mathbb{Z}$ by letting $\widehat{\xi}(t)=\overline{0}$. Observe that $M_{n}=\operatorname{ker}(\widehat{\xi})$ is the normal closure of $N_{n}$ and $t$ in $L_{n}$, and satisfies

$$
\left|L_{n}: M_{n}\right|=2 \text { and } M_{n} \cap G_{n}=N_{n} .
$$

We shall now check that conditions (3.4) and (3.5) are satisfied for $i=n$, when $G_{n}$ is replaced by $L_{n}$ and $N_{n}$ is replaced by $M_{n}$. Note that the associated subgroups $\left(\left\langle a_{n}\right\rangle\right.$ and $\langle d\rangle$ in Case 1 , and $\langle e\rangle$ and $\langle d\rangle$ in Case 2) of the HNN-extension $L_{n}$ are malnormal in $G_{n}$ by Lemma 2.4, and their $G_{n}$-conjugates can only intersect trivially. Therefore any malnormal cyclic subgroup of $G_{n}$ will remain malnormal in $L_{n}$ by part (a) of Lemma 2.11. It follows that

$$
\mathrm{C}_{L_{n}}\left(a_{n}^{l}\right)=\left\langle a_{n}\right\rangle, \forall l \in \mathbb{Z} \backslash\{0\}, \text { and } \mathrm{C}_{L_{n}}(u)=\mathrm{C}_{G_{n}}(u) \subseteq H_{n}, \forall u \in H_{n} \backslash\left\langle a_{n}^{2}\right\rangle^{H_{n}} .
$$

Assume that for some $l \in 2 \mathbb{N}, a_{n}^{l}$ is conjugate in $L_{n}$ to an element $h \in H_{n}$. We will show that these two elements are actually conjugate in $G_{n}$. Arguing by contradiction, suppose that $h \notin\left(a_{n}^{l}\right)^{G_{n}}$. If $h \in\left(a_{n}^{k}\right)^{G_{n}}$, for $k \neq l$, then, since $\left\langle a_{n}\right\rangle$ is malnormal in $L_{n}, a_{n}^{k}$ cannot be conjugate to $a_{n}^{l}$ in $L_{n}$, contradicting our assumption. Thus $h \notin\left\langle a_{n}\right\rangle^{G_{n}}$. If we are in Case 1 above, the latter, combined with (3.8), implies that $a_{n}^{l} \notin h^{L_{n}}$ by part (b) of Lemma 2.11, which again contradicts the assumption that $h$ is conjugate to $a_{n}^{l}$ in $L_{n}$. Similarly, in Case 2, in view of (3.10) and the assumption that $a_{n} \stackrel{G_{n}}{\not} d$, part (b) of Lemma 2.11 shows that $h \notin\left(a_{n}^{l}\right)^{L_{n}}$, leading to another contradiction. Thus we have shown that $a_{n}^{l} \in h^{G_{n}}$. After recalling (3.5), we see that the following has been established:

$$
\text { if } a_{n}^{l} \in h^{L_{n}} \text {, for some } l \in 2 \mathbb{N} \text { and } h \in H_{n} \text {, then } a_{n}^{l} \in h^{H_{n}} \text {. }
$$

We will now construct the group $G_{n+1}$ as a quotient of $L_{n}$, using Theorem 2.6. Denote $F=$ $\left\langle a_{n}, b_{n}^{c}\right\rangle \cap N_{n} \leqslant G_{n}$ and observe that $\left|\left\langle a_{n}, b_{n}^{c}\right\rangle: F\right|=2$. Note that the subgroup $\left\langle a_{n}^{2}, b_{n}^{c}\right\rangle \leqslant F$ is non-elementary (otherwise $a_{n}^{l}=\left(b_{n}^{k}\right)^{c}$, for some $l \in 2 \mathbb{N}, k \in \mathbb{Z} \backslash\{0\}$, which, by (3.5), would imply that $a_{n}^{l} \in\left(b_{n}^{k}\right)^{H_{n}}$, contradicting the fact that $a_{n}^{2}$ and $b_{n}$ freely generate $H_{n}$ by (3.3)). Therefore $F$ is non-elementary.

Let us prove that $Q_{n}=H_{n} \cup H_{n}^{a_{n}}$ is small relative to $F$ in $L_{n}$. Indeed, by Theorem 2.7, it is sufficient to show that $\left|F:\left(F \cap H_{n}^{x}\right)\right|=\infty$ for all $x \in L_{n}$. Arguing by contradiction, assume that

$$
\left|F:\left(F \cap H_{n}^{x}\right)\right|<\infty \text { for some } x \in L_{n} .
$$

Since $a_{n}^{2} \in F$, by (3.15) there must exist $l \in 2 \mathbb{N}$ and $h \in H_{n}$ such that $a_{n}^{l}=h^{x}$. So, in view of (3.14), we can find $y \in H_{n}$ satisfying $a_{n}^{l}=h^{y}$. The latter yields that $h=y^{-1} a_{n}^{l} y=x^{-1} a_{n}^{l} x$, so that $x y^{-1} \in \mathrm{C}_{L_{n}}\left(a_{n}^{l}\right)=\left\langle a_{n}\right\rangle$ by (3.13). Hence $x=a_{n}^{k} y$, for some $k \in \mathbb{Z}$, and $H_{n}^{x}=a_{n}^{k} H_{n} a_{n}^{-k}$. Since $F$ is normalized by $a_{n}$, by definition, the latter, combined with (3.15), implies that $\left|F:\left(F \cap H_{n}\right)\right|<\infty$ and $\left|F:\left(F \cap H_{n}^{a_{n}}\right)\right|<\infty$, hence

$$
\left|F:\left(F \cap\left(H_{n} \cap H_{n}^{a_{n}}\right)\right)\right|<\infty .
$$

But, by the injectivity of $\psi_{n}$ on $Q_{0}$ (3.3) and part (ii) of Lemma 2.2, we have

$$
H_{n} \cap H_{n}^{a_{n}}=\psi_{n}\left(H_{0}\right) \cap \psi_{n}\left(H_{0}^{a_{0}}\right)=\psi_{n}\left(H_{0} \cap H_{0}^{a_{0}}\right)=\psi_{n}\left(\left\langle a_{0}^{2}\right\rangle\right)=\left\langle a_{n}^{2}\right\rangle .
$$


Thus (3.16) yields $\left|F:\left(F \cap\left\langle a_{n}^{2}\right\rangle\right)\right|<\infty$, contradicting the fact that $F$ is a non-elementary subgroup of $G_{n}$. Hence we have proved that $Q_{n}$ is small relative to $F$ in $L_{n}$.

Therefore we can apply Theorem 2.6 to $L_{n}, F, Q_{n}$ and $U=\left\{b_{n}, t\right\}$. Let $w_{1}, w_{2} \in F$ be the elements from the claim of this theorem, let $K_{n}$ denote the normal closure of the elements $b_{n} w_{1}$ and $t w_{2}$ in $L_{n}$, let $G_{n+1}=L_{n} / K_{n}$, let $\eta_{n+1}: L_{n} \rightarrow G_{n+1}$ be the natural epimorphism and $\phi_{n+1}: G_{n} \rightarrow G_{n+1}$ be the restriction of $\eta_{n+1}$ to $G_{n} \leqslant L_{n}$. Denote $\psi_{n+1}=\phi_{n+1} \circ \psi_{n}: G_{0} \rightarrow G_{n+1}$, $a_{n+1}=\phi_{n+1}\left(a_{n}\right)=\psi_{n+1}\left(a_{0}\right), b_{n+1}=\phi_{n+1}\left(b_{n}\right)=\psi_{n+1}\left(b_{0}\right)$, etc., as in the beginning of the proof, for $i=n+1$. Then the following conditions will be satisfied by Theorem 2.6.

(a) $G_{n+1}$ is a torsion-free hyperbolic group. Moreover, since $L_{n}=\left\langle a_{n}, b_{n}, t\right\rangle$ and $\eta_{n+1}(t)=$ $\eta_{n+1}\left(w_{2}^{-1}\right) \in \eta_{n+1}\left(G_{n}\right)$, we see that $\eta_{n+1}\left(G_{n}\right)=G_{n+1}$, i.e., $\phi_{n+1}: G_{n} \rightarrow G_{n+1}$ is surjective and $G_{n+1}=\left\langle a_{n+1}, b_{n+1}\right\rangle$. Hence (3.1) holds for $i=n+1$.

(b) The restriction of $\phi_{n+1}$ to $Q_{n}$ is injective and $H_{n+1}=\phi_{n+1}\left(H_{n}\right), Q_{n+1}=\phi_{n+1}\left(Q_{n}\right)$ are quasiconvex in $G_{n+1}$. Thus (3.3) holds for $i=n+1$.

(c) For all $x \in H_{n}, \mathrm{C}_{G_{n+1}}\left(\eta_{n+1}(x)\right)=\eta_{n+1}\left(\mathrm{C}_{L_{n}}(x)\right)$. In view of (3.13) and the injectivity of $\eta_{n+1}$ on $H_{n}$, this implies that (3.4) holds for $i=n+1$.

(d) $\eta_{n+1}$ preserves conjugacy on $H_{n}$. In view of (3.14), this implies that (3.5) holds for $i=n+1$.

Observe that $b_{n+1}=\phi_{n+1}\left(b_{n}\right)=\phi_{n+1}\left(w_{1}^{-1}\right) \in \phi_{n+1}(F)$ in $G_{n+1}$. Since $F \subset\left\langle a_{n}, b_{n}^{c}\right\rangle$ and $G_{n+1}=\left\langle a_{n+1}, b_{n+1}\right\rangle$, we can conclude that $G_{n+1}=\phi_{n+1}\left(\left\langle a_{n}, b_{n}^{c}\right\rangle\right)=\left\langle a_{n+1}, b_{n+1}^{g_{n+1}}\right\rangle$, where $g_{n+1}=$ $\phi_{n+1}(c)=\psi_{n+1}\left(f_{n+1}\right)$. Thus (3.7) holds for $i=n+1$, and it remains to check that $G_{n+1}$ satisfies (3.2) and (3.6).

Note that $N_{n+1}=\phi_{n+1}\left(N_{n}\right)$ is a normal subgroup in $G_{n+1}$ of index at most 2 because $\phi_{n+1}$ is surjective and $\left|G_{n}: N_{n}\right|=2$. Obviously $N_{n+1} \subseteq \eta_{n+1}\left(M_{n}\right)$. The opposite inclusion follows from the fact that $M_{n}$ is generated, as a normal subgroup of $L_{n}$, by $N_{n}$ and $t$, and

$$
\eta_{n+1}(t)=\eta_{n+1}\left(w_{2}^{-1}\right) \in \eta_{n+1}(F) \subseteq \eta_{n+1}\left(N_{n}\right)=N_{n+1}
$$

Hence $N_{n+1}=\eta_{n+1}\left(M_{n}\right)$ in $L_{n}$. Recall that, by construction, $b_{n}, t \in M_{n}$ and $w_{1}, w_{2} \in F \subset M_{n}$ in $L_{n}$, yielding that ker $\eta_{n+1}=K_{n} \subseteq M_{n}$. Therefore $G_{n+1} / N_{n+1} \cong L_{n} / M_{n} \cong \mathbb{Z} / 2 \mathbb{Z}$, i.e., $N_{n+1}$ has index 2 in $G_{n+1}$. It follows that condition (3.2) holds for $i=n+1$.

By construction, $\psi_{n}\left(f_{n+1}\right)=c$ either belongs to $\left\langle a_{n}\right\rangle^{t}$ (Case 1) or to $H_{n}^{t}$ (Case 2) in $L_{n}$. Therefore condition (3.6) for $i=n+1$ follows from (3.17). This concludes the inductive step in our argument, and thus finishes the construction of a sequence of hyperbolic groups $G_{0}, G_{1}, G_{2}, \ldots$, together with epimorphisms $\phi_{i}: G_{i-1} \rightarrow G_{i}, i \in \mathbb{N}$, satisfying properties (3.1)-(3.7) above.

We can now define the group $G$ as the direct limit of the sequence $\left(G_{i-1}, \phi_{i}\right)_{i \in \mathbb{N}}$. In other words, $G=G_{0} / K$, where $K=\bigcup_{i=1}^{\infty} \operatorname{ker} \psi_{i}$. Let $\psi: G_{0} \rightarrow G$ be the natural epimorphism, so that $\operatorname{ker} \psi=K$ and $\psi$ factors through $\psi_{i}: G_{0} \rightarrow G_{i}$, for each $i \in \mathbb{N}$.

Let $a=\psi\left(a_{0}\right), b=\psi\left(b_{0}\right), H=\psi\left(H_{0}\right)=\left\langle a^{2}, b\right\rangle \leqslant G$ and $N=\psi\left(N_{0}\right)=\left\langle a^{2}, b, b^{a}\right\rangle \triangleleft G$. We will now check that $G, H$ and $N$ satisfy the properties from the claim of Theorem 1.3.

The group $G$ is torsion-free as a direct limit of torsion-free groups $G_{i}$. The index $|G: N|=2$ because, by (3.2), ker $\psi_{i} \subseteq N_{0}$ for all $i \in \mathbb{N}$. By (3.3), each $\psi_{i}$ is injective on $Q_{0}$, hence the same is true for $\psi$. It follows that $H \cap H^{a}=\psi\left(H_{0} \cap H_{0}^{a_{0}}\right)=\left\langle a^{2}\right\rangle$ (by Lemma 2.2), and $H \cong H_{0}$ is freely generated by $a^{2}$ and $b$. If $|N: H|<\infty$ then $\left|N: H^{a}\right|<\infty$, since $a$ normalizes $N$, hence $\left|N:\left(H \cap H^{a}\right)\right|<\infty$, which would mean that $N$ is virtually cyclic, contradicting the fact that it contains a non-abelian free subgroup $H$. Therefore we can deduce that $|N: H|=\infty$.

Given any $x, y \in G$, the subgroup $\left\langle a^{x}, b^{y}\right\rangle$ is conjugate to the subgroup $\left\langle a, b^{g}\right\rangle$, where $g=x^{-1} y$. By construction, $g=\psi\left(f_{i}\right)$, for some $i \in \mathbb{N} \cup\{0\}$, and (3.7) implies that $G_{i}$ is generated by $a_{i}=\psi_{i}\left(a_{0}\right)$ and $b_{i}^{g_{i}}=\psi_{i}\left(b_{0}^{f_{i}}\right)$. It follows that $G=\left\langle a, b^{g}\right\rangle$, since $\psi$ factors through $\psi_{i}$. Hence $G=\left\langle a^{x}, b^{y}\right\rangle$ and $\{a, b\}$ is an invariable generating set for $G$. 
It remains to show that every element of $N$ is conjugate to an element of $H$ in $N$. Indeed, (3.6) easily implies that $G=H^{N} \cup\langle a\rangle^{N}$, and since

$$
\langle a\rangle^{N} \cap N=(\langle a\rangle \cap N)^{N}=\left\langle a^{2}\right\rangle^{N} \subset H^{N},
$$

we can conclude that $N=H^{N}$, as required. This finishes the proof of Theorem 1.3.

Remark 3.1. Let $G$ be the group constructed in the proof of Theorem 1.3. Then centralizers of non-trivial elements in $G$ are cyclic.

Indeed, for any $g \in G \backslash\{1\}, g$ has infinite order and $g^{2} \in N$, so, by claim (iv) of the theorem, $g^{2}$ is conjugate to some element $u \in H \backslash\{1\}$. If $\mathrm{C}_{G}(u) \subseteq H$, then $\mathrm{C}_{G}(u)=\mathrm{C}_{H}(u)$ is cyclic, as $H$ is free. Otherwise, (3.4) implies that $u \in\left\langle a^{2}\right\rangle^{H}$ and $\mathrm{C}_{G}(u)$ is conjugate to $\langle a\rangle$. Thus $\mathrm{C}_{G}(u)$ is cyclic, so the same is true for $\mathrm{C}_{G}\left(g^{2}\right)$. Therefore $\mathrm{C}_{G}(g)$ must also be cyclic, as a subgroup of $\mathrm{C}_{G}\left(g^{2}\right)$ (in fact, in this case $\mathrm{C}_{G}(g)=\mathrm{C}_{G}\left(g^{2}\right)$ ).

Remark 3.2. The construction of the group $G$ and its index two subgroup $N$ in Theorem 1.3 is fairly flexible, and many additional properties can be achieved:

- by adding a sufficiently large finite subset of $G_{i}$ to $Q_{i}$ at each step, one can ensure that $G$ is lacunary hyperbolic (see [22] for the definition and properties of lacunary hyperbolic groups);

- by modifying $N_{0}$ and $G_{0}$ with the help of Theorem 2.6, one can arrange $N$ to be a quotient of any given torsion-free non-elementary hyperbolic group; in particular, $N$ and $G$ can be made to satisfy Kazhdan's property (T);

- by changing the construction at each step, one can achieve even more and ensure that $N$ is a common quotient of all non-cyclic torsion-free hyperbolic groups;

- it should be possible to extend the method of proof to produce examples of FIG groups with non-IG subgroups of index $k$, for every $k \geq 2$, though additional technical modifications will be necessary.

\section{Finitely generated IG groups that are not FiG}

Proof of Theorem 1.4. As before, the desired group $G$ will be constructed as a direct limit of hyperbolic groups $G_{i}, i \geq 0$. Let $G_{0}$ be the free group of rank 3 , freely generated by $\left\{a_{0}, b_{0}, c_{0}\right\}$, and set $Q_{0}=H_{00}=\{1\} \leqslant G_{0}$. Let $S_{0}=\emptyset, S_{1}=\{1\}, S_{2}, \ldots$ be an enumeration of all finite subsets of $G_{0}$, let $Y_{0}=\{1\}, Y_{1}=\left\langle a_{0}\right\rangle, Y_{2}=\left\langle a_{0}, b_{0}\right\rangle, \ldots$ be an enumeration of all finitely generated subgroups of $G_{0}$.

Let $G_{1}$ be a copy of $G_{0}$, with a fixed isomorphism $\phi_{1}: G_{0} \rightarrow G_{1}$. Define $H_{10}=\phi_{1}\left(H_{00}\right)=\{1\}$, $H_{11}=\left\langle b_{1}, c_{1}\right\rangle$, where $b_{1}=\phi_{1}\left(b_{0}\right) \in G_{1}, c_{1}=\phi_{1}\left(c_{0}\right) \in G_{1}$, and let $Q_{1}=H_{10} \cup H_{11}=\phi_{1}\left(Q_{0}\right) \cup$ $H_{11} \subset G_{1}$.

Now suppose that for some $n \geq 1$ and each $i=1, \ldots, n$ we have already constructed a group $G_{i}$, an epimorphism $\phi_{i}: G_{i-1} \rightarrow G_{i}$, and subgroups $H_{i j} \leqslant G_{i}, j=0,1, \ldots, i$, such that $H_{i j}=$ $\phi_{i}\left(H_{i-1, j}\right)$, whenever $j \in\{0, \ldots, i-1\}$. We let $\psi_{i}: G_{0} \rightarrow G_{i}$ be the epimorphism defined by $\psi_{i}=\phi_{i} \circ \cdots \circ \phi_{1}$, set $T_{i}=\psi_{i}\left(S_{i}\right) \subset G_{i}, Z_{i}=\psi_{i}\left(Y_{i}\right) \leqslant G_{i}$, and $Q_{i}=\bigcup_{j=0}^{i} H_{i j}=\phi_{i}\left(Q_{i-1}\right) \cup H_{i i} \subseteq G_{i}$.

Arguing by induction, we assume that the following conditions hold for every $i=1, \ldots, n$ :

(4.1) $G_{i}$ is a non-elementary torsion-free hyperbolic group;

(4.2) $\phi_{i}$ is injective on $Q_{i-1}$, and $H_{i j}$ is free and quasiconvex in $G_{i}, j=0, \ldots, i$;

(4.3) $\phi_{i}$ preserves conjugacy and centralizers on $Q_{i-1}$;

(4.4) $H_{i j}$ is malnormal in $G_{i}$, for all $j=0, \ldots, i$; 
(4.5) for all $g \in G_{i}, 0 \leq j<k \leq i$, the intersection $H_{i j}^{g} \cap H_{i k}$ is cyclic;

$\left|G_{i}: H_{i i}\right|=\infty$ and there exists $x \in H_{i i} \backslash\{1\}$ such that $\langle x\rangle \cap\left(\bigcup_{j=1}^{i-1} H_{i j}^{G_{i}}\right)=\{1\}$

(4.7) $T_{i} \subseteq H_{i i}^{G_{i}}$

(4.8) one of the following conditions is satisfied:

- $Z_{i}=G_{i}$, or

- $Z_{i}$ is cyclic, or

- $\left|Z_{i}:\left(Z_{i} \cap H_{i j}^{u}\right)\right|<\infty$ for some $j=0,1, \ldots, i$, and some $u \in G_{i}$.

Note that for $i=1$ the group $G_{1}$, the epimorphism $\phi_{1}: G_{0} \rightarrow G_{1}$, and the subgroups $H_{10}, H_{11} \leqslant$ $G_{1}$ satisfy all of the above properties by definition, so the base of induction has been established. Our aim now is to construct a group $G_{n+1}$, an epimorphism $\phi_{n+1}: G_{n} \rightarrow G_{n+1}$ and a subgroup $H_{n+1, n+1} \leqslant G_{n+1}$, enjoying properties (4.1)-(4.8) for $i=n+1$. As before, our method involves an intermediate torsion-free hyperbolic group $L_{n}$ and a free quasiconvex subgroup $X \leqslant L_{n}$, such that $G_{n} \leqslant L_{n}$ and $\psi_{n}\left(S_{n+1}\right)$ is pointwise conjugate into $X$ in $L_{n}$. The group $G_{n+1}$ will be obtained as a small cancellation quotient of $L_{n}$, and the subgroup $H_{n+1, n+1} \leqslant G_{n+1}$ will be defined as the image of $X$ in $G_{n+1}$.

Recall that $\psi_{n}: G_{0} \rightarrow G_{n}$ is the epimorphism $\phi_{n} \circ \cdots \circ \phi_{1}$, and suppose that $\psi_{n}\left(S_{n+1}\right) \backslash$ $\{1\}=\left\{s_{1}, \ldots, s_{l}\right\} \subset G_{n}$, for some $l \in \mathbb{N} \cup\{0\}$. Let $d_{1}, \ldots, d_{l} \in G_{n}$ be the elements satisfying $\mathrm{C}_{G_{n}}\left(s_{k}\right)=\left\langle d_{k}\right\rangle$, for $k=1, \ldots, l$ (cf. Lemma 2.4). Let $X$ be a free group of rank $l+1$, with free basis $\left\{x_{0}, x_{1}, \ldots, x_{l}\right\}$. We define the group $L_{n}$ by the following presentation:

$$
L_{n}=\left\langle G_{n}, x_{0}, \ldots, x_{l}, t_{1}, \ldots, t_{l} \| t_{k} x_{k} t_{k}^{-1}=d_{k}, k=1, \ldots, l\right\rangle .
$$

Since $G_{n}$ is torsion-free, each of $d_{1}, \ldots, d_{l}$ has infinite order in $G_{n}$, so $L_{n}$ is an $l$-fold HNNextension of the free product $G_{n} * X$ with associated cyclic subgroups. The group $G_{n} * X$ is hyperbolic, as a free product of hyperbolic groups, and $G_{n}, X$ are both quasiconvex in it (for example, by [18, Lemma 1.2]). After applying Lemma $2.10 l$ times, we see that $L_{n}$ is a torsion-free hyperbolic group and $G_{n}, X$ are quasiconvex in it. Therefore $H_{n j}$ is a quasiconvex subgroup of $L_{n}$, for each $j=0, \ldots, n$, by part (5) of Remark 2.3 .

Now, $G_{n}$ and $X$ are malnormal in $G_{n} * X$, being free factors, hence $\left\langle d_{k}\right\rangle$ and $H_{n j}$ are malnormal in $G_{n} * X$, for all $k=1, \ldots, l$ and $j=0, \ldots, n$, by (4.4). Observe that for any group $A$ and any cyclic group $\langle x\rangle,\langle x\rangle$ and every malnormal subgroup of $A$ are malnormal in the free product $A *\langle x\rangle$; moreover, no non-trivial element of $A$ is conjugate to an element of $\langle x\rangle$. Therefore we can apply claim (a) of Lemma $2.11 l$ times to show that $H_{n j}$ and $X$ are malnormal in $L_{n}$, for every $j=0, \ldots, n$. It follows that $\mathrm{C}_{L}(h) \subseteq H_{n j}$ for all $h \in H_{n j} \backslash\{1\}, j=0, \ldots, n$; in particular,

$$
\mathrm{C}_{L}(h)=\mathrm{C}_{G_{n}}(h) \text {, for every } h \in Q_{n} \backslash\{1\} \text {. }
$$

From the presentation (4.9) it is easy to see that there is a retraction $\rho: L_{n} \rightarrow G_{n}$, such that the restriction of $\rho$ to $G_{n}$ is the identity map, $\rho\left(x_{0}\right)=1, \rho\left(x_{k}\right)=d_{k}$ and $\rho\left(t_{k}\right)=1$ for all $k=1, \ldots, l$. It follows that

(4.11) two elements of $G_{n}$ are conjugate in $L_{n}$ if and only if they are conjugate in $G_{n}$.

For the next part it will be more convenient to think of $L_{n}$ as the fundamental group of a graph of groups with two vertices $v_{1}, v_{2}$ and $l+1$ edges $e_{0}, \ldots, e_{l}$, joining these two vertices: see Figure 1 . The vertex groups in this splitting will be $G_{n}$ for $v_{1}$ and $X$ for $v_{2}$, the edge group for $e_{0}$ will be trivial, and the edge groups for $e_{1}, \ldots, e_{l}$ will be infinite cyclic. This gives rise to an action of $L_{n}$ 


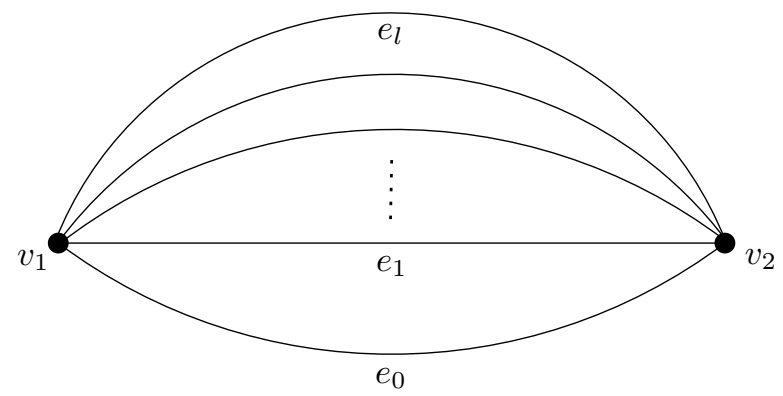

FIgURE 1. The underlying graph for the splitting of $L_{n}$.

on the Basse-Serre tree $\mathcal{T}$ corresponding to this splitting. Vertex stabilizers for this action are conjugates of $G_{n}$ or $X$ in $L_{n}$ and edge stabilizers are cyclic. Hence the intersection of stabilizers of two distinct vertices of $\mathcal{T}$ is cyclic, which immediately yields the following observations:

$$
G_{n}^{u} \cap X \text { is cyclic, for all } u \in L_{n} \text {, and }
$$

$$
\text { if } w \in L_{n} \backslash G_{n} \text { then } G_{n}^{w} \cap G_{n} \text { is cyclic. }
$$

The last observation, combined with (4.5), implies

$$
\text { for all } u \in L_{n}, 0 \leq j<k \leq n \text {, the intersection } H_{n j}^{u} \cap H_{n k} \text { is cyclic. }
$$

Let $Q=Q_{n} \cup X=\bigcup_{j=1}^{n} H_{n j} \cup X \subset L_{n}$ and $Z=\psi_{n}\left(Y_{n+1}\right) \leqslant G_{n}$. If $Z$ is non-elementary and $Q$ is small relative to $Z$ in $L_{n}$, then set $F=Z$. Otherwise, set $F=G_{n}$. Recall that $G_{n}$ is torsion-free and non-elementary (by (4.1)), hence (4.6) implies that $\left|G_{n}: H_{n j}\right|=\infty$, for each $j=0, \ldots, n$. Combined with (4.13), this yields that $\left|G_{n}:\left(G_{n} \cap w^{-1} H_{n j} w\right)\right|=\left|G_{n}^{w}:\left(G_{n}^{w} \cap H_{n j}\right)\right|=\infty$ for all $w \in L_{n}$ and $j=0, \ldots, n$. On the other hand, $\left|G_{n}:\left(G_{n} \cap u^{-1} X u\right)\right|=\infty$ for all $u \in L_{n}$ by (4.12). Therefore we can apply Theorem 2.7 to conclude that $Q$ is small relative to $G_{n}$ in $L_{n}$. Thus, in any case, $F$ is non-elementary, $Q$ is small relative to $F$ in $L_{n}$ and $F \subseteq G_{n}$.

Since $Q$ is quasiconvex in $L_{n}$ (by part (4) of Remark 2.3), we can apply Theorem 2.6 to $L_{n}$, $F, Q$ and $U=\left\{x_{0}, \ldots, x_{l}, t_{1}, \ldots, t_{l}\right\}$. Let $G_{n+1}$ denote the resulting quotient of $L_{n}$, let $\eta_{n+1}$ : $L_{n} \rightarrow G_{n+1}$ be the natural epimorphism, which identifies each element of $U$ with some element of $F$ in $G_{n+1}$. Since $F \subseteq G_{n}$ and $L_{n}=\left\langle G_{n}, U\right\rangle$, we see that $G_{n+1}=\eta_{n+1}\left(L_{n}\right)=\eta_{n+1}\left(G_{n}\right)$, i.e., the restriction $\phi_{n+1}: G_{n} \rightarrow G_{n+1}$, of $\eta_{n+1}$ to $G_{n}$, is surjective. Set $H_{n+1, n+1}=\eta_{n+1}(X) \leqslant G_{n+1}$, $H_{n+1, j}=\phi_{n+1}\left(H_{n j}\right) \leqslant G_{n+1}$ if $0 \leq j \leq n$, and $Q_{n+1}=\bigcup_{j=0}^{n+1} H_{n+1, j}$. Let $\psi_{n+1}=\phi_{n+1} \circ \psi_{n}$ : $G_{0} \rightarrow G_{n+1}, T_{n+1}=\psi_{n+1}\left(S_{n+1}\right) \subset G_{n+1}, Z_{n+1}=\psi_{n+1}\left(Y_{n+1}\right) \leqslant G_{n+1}$.

Theorem 2.6 implies that all of the following hold.

(a) $G_{n+1}$ is a non-elementary torsion-free hyperbolic group, so (4.1) is true for $i=n+1$.

(b) The restriction of $\eta_{n+1}$ to $Q=Q_{n} \cup X$ is injective, and $H_{n+1, j}$ is a quasiconvex subgroup of $G_{n+1}$, for all $j=0, \ldots, n+1$. It follows that $\phi_{n}$ is injective on $Q_{n}$, and $H_{n+1, j} \cong H_{n j}$, $0 \leq j \leq n, H_{n+1, n+1} \cong X$ are free subgroups of $G_{n+1}$. Thus (4.2) holds for $i=n+1$.

(c) $\eta_{n+1}$ preserves centralizers on $Q$, hence, by (4.10), $\phi_{n+1}$ preserves centralizers on $Q_{n}$.

(d) $\eta_{n+1}$ preserves conjugacy on $Q$ :

$$
\text { if } g, h \in Q_{n} \cup X \text {, then } \eta_{n+1}(h) \in \eta_{n+1}(g)^{G_{n+1}} \text { in } G_{n+1} \text { implies } h \in g^{L_{n}} \text { in } L_{n} \text {. }
$$

Combined with (4.11), this immediately shows that $\phi_{n+1}$ preserves conjugacy on $Q_{n}$.

Claims (c) and (d) above imply that (4.3) holds for $i=n+1$. We can also apply Lemma 2.8 to deduce that for every $j=0, \ldots, n+1, H_{n+1, j}$ is malnormal in $G_{n+1}$, i.e., (4.4) is satisfied for $i=n+1$. 
The fact that (4.5) is satisfied in $G_{n+1}$ follows from Lemma 2.9. Indeed, for any $0 \leq j<k \leq$ $n+1, H_{n+1, j}$ and $H_{n+1, k}$ are images, under $\eta_{n+1}$, of subgroups contained in $Q_{n} \cup X$ in $L_{n}$, so the assumptions of Lemma 2.9 are satisfied by (4.15), (4.14) and (4.12).

By the above argument, $H_{n+1, n+1} \cap H_{n+1,1}$ is cyclic, and since $H_{n+1,1} \cong H_{11}$ (by (4.2)) is a free group of rank 2 , we can immediately deduce that $H_{n+1, n+1}$ must have infinite index in $G_{n+1}$. Now, since the generator $x_{0}$, of $X$, does not participate in any of the defining relations from (4.9), we see that $L_{n} \cong B *\left\langle x_{0}\right\rangle$, where $B=\left\langle G_{n}, x_{1}, \ldots, x_{l}, t_{1}, \ldots, t_{l}\right\rangle \leqslant L_{n}$. In particular, $\left\langle x_{0}\right\rangle \cap H_{n j}^{L_{n}}=\{1\}$, for all $j=0, \ldots, n$, which can be combined with (4.15) to give $\left\langle\eta_{n+1}\left(x_{0}\right)\right\rangle \cap H_{n+1, j}^{G_{n+1}}=\{1\}$, for all $j=0, \ldots, n$. As $\eta_{n+1}\left(x_{0}\right)$ is an infinite order element of $\eta_{n+1}(X)=H_{n+1, n+1}$, we can conclude that (4.6) is satisfied for $i=n+1$.

The inclusion (4.7) for $i=n+1$ is an immediate consequence of the construction of $L_{n}$ as the HNN-extension (4.9), and it remains to verify that $G_{n+1}$ satisfies (4.8) for $i=n+1$, where $Z_{n+1}=\psi_{n+1}\left(Y_{n+1}\right)=\eta_{n+1}(Z)$. But this is indeed the case due to our choice of the subgroup $F \leqslant G_{n}$ above and Theorem 2.7.

Thus we have checked that $G_{n+1}, \phi_{n+1}: G_{n} \rightarrow G_{n+1}$ and $H_{n+1, n+1} \leqslant G_{n+1}$ satisfy all of the properties (4.1)-(4.8) for $i=n+1$, which completes our inductive construction.

Let $G$ be the direct limit of the sequence $\left(G_{i-1}, \phi_{i}\right)_{i \in \mathbb{N}}$. Then $G$ can be described as the quotient of $G_{0}$ by $K=\bigcup_{i=1}^{\infty}$ ker $\psi_{i}$, and the natural epimorphism $\psi: G_{0} \rightarrow G$ factors through $\psi_{i}: G_{0} \rightarrow G_{i}$, for each $i \in \mathbb{N}$. Let $\xi_{i}: G_{i} \rightarrow G$ denote the resulting epimorphism such that $\psi=\xi_{i} \circ \psi_{i}$, for each $i \in \mathbb{N}$. A commutative diagram involving epimorphisms between the groups $G_{0}, G_{i}, G_{i+1}$ and $G$, $i \in \mathbb{N}$, discussed so far, is depicted in Figure 2 below.

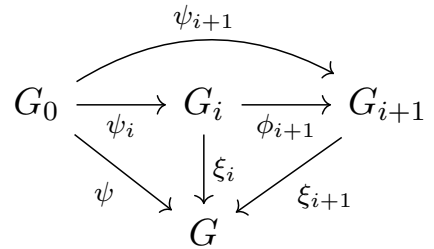

Figure 2. A commutative diagram of maps between $G_{0}, G_{i}, G_{i+1}$ and $G$.

Lemma 4.1. For all $j \geq 0$ the epimorphism $\xi_{j}: G_{j} \rightarrow G$ preserves conjugacy on $Q_{j}$.

Proof of Lemma 4.1. Suppose $\xi_{j}(h)=\xi_{j}(g)^{q}$, for some $g, h \in Q_{j}$ and some $q \in G$. Let $g_{0}, h_{0}, q_{0} \in$ $G_{0}$ be arbitrary preimages of the elements $g, h, q$ in $G_{0}$, so that $g=\psi_{j}\left(g_{0}\right), h=\psi_{j}\left(h_{0}\right)$ and $q=\psi\left(q_{0}\right)$. Then $h_{0}^{-1} g_{0}^{q_{0}} \in \operatorname{ker} \psi=\bigcup_{n=1}^{\infty} \operatorname{ker} \psi_{n}$, so $h_{0}^{-1} g_{0}^{q_{0}} \in \operatorname{ker} \psi_{i}$, for some $i \in \mathbb{N}$. Moreover, we can assume that $i>j$, as ker $\psi_{i} \subseteq \operatorname{ker} \psi_{i+1}$ for all $i$. Hence $g^{\prime}=\psi_{i}\left(g_{0}\right)$ is conjugate to $h^{\prime}=\psi_{i}\left(h_{0}\right)$ in $G_{i}$. Let $\varphi: G_{j} \rightarrow G_{i}$ be the epimorphism defined by $\varphi=\phi_{i} \circ \cdots \circ \phi_{j+1}$, and observe that $g^{\prime}=\varphi(g)$ and $h^{\prime}=\varphi(h)$, so that $g^{\prime}, h^{\prime} \in \varphi\left(Q_{j}\right)$ in $G_{i}$. Since $h^{\prime} \in\left(g^{\prime}\right)^{G_{i}}$ we can apply (4.3) $i-j$ times to conclude that $h \in g^{G_{j}}$ in $G_{j}$, as required.

The next statement also follows from (4.3) and can be proved similarly to Lemma 4.1.

Lemma 4.2. For all $j \geq 0$ the epimorphism $\xi_{j}: G_{j} \rightarrow G$ preserves centralizers on $Q_{j}$.

We can now start checking that $G$ satisfies all the properties listed in the statement of Theorem 1.4. The group $G$ is indeed 2-generated, because it is a quotient of $G_{2}$, and $G_{2}=Z_{2}$ is a quotient of $Z=\left\langle a_{1}, b_{1}\right\rangle=\phi_{1}\left(Y_{2}\right)$, by construction ( $Z$ is non-elementary and $Q_{1}=\left\langle b_{1}, c_{1}\right\rangle$ is small relative to $Z$ in $L_{1}$, as $\left\{a_{1}, b_{1}, c_{1}\right\}$ is a free generating set of $G_{1}$ ). Since $G_{i}$ is torsion-free, for each $i \in \mathbb{N}, G$ is also torsion-free. 
Observe that $\xi_{j}\left(H_{j j}\right)=\xi_{i}\left(H_{i j}\right)$ in $G$, whenever $0 \leq j \leq i$, and denote this subgroup $H_{j} \leqslant G$. The epimorphism $\xi_{j}$ is injective on $H_{j j}$ by (4.2), so $H_{j} \cong H_{j j}$ is finitely generated and free. Moreover, in view of (4.4) and Lemmas 4.1, 4.2, we can apply Lemma 2.8 to conclude that $H_{j}$ is malnormal in $G$. Thus claim (i) of Theorem 1.4 has been established.

Now, by (4.6), for each $i \in \mathbb{N}$ there is $x \in H_{i i} \backslash\{1\}$ such that $\langle x\rangle \cap H_{i j}^{G_{i}}=\{1\}$ in $G_{i}$, whenever $0 \leq j<i$. Set $r=\xi_{i}(x) \in H_{i}$, then $r \neq 1$ in $G$, by $(4.2)$, and $\langle r\rangle \cap H_{j}^{G}=\{1\}$, provided $0 \leq j<i$, by Lemma 4.1. This proves claim (ii) of Theorem 1.4.

If $S$ is an arbitrary finite subset of $G$, then $S=\psi\left(S_{i}\right)$, for some $i \in \mathbb{N} \cup\{0\}$, and $T_{i}=\psi_{i}\left(S_{i}\right)$ is pointwise conjugate into $H_{i i}$ in $G_{i}$, by (4.7). Consequently, $S=\xi_{i}\left(T_{i}\right)$ is pointwise conjugate into the subgroup $H_{i}=\xi_{i}\left(H_{i i}\right)$ in $G$, which establishes claim (iii) of Theorem 1.4.

Claim (iv) of the theorem follows from the lemma below.

Lemma 4.3. For all $i, j \geq 0, i \neq j$, and all $h \in G$, the intersection $H_{j}^{h} \cap H_{i}$ is cyclic in $G$.

Proof of Lemma 4.3. Without loss of generality, suppose that $j<i$. By construction, $H_{j}=$ $\xi_{i}\left(H_{i j}\right)$ and $H_{i}=\xi_{i}\left(H_{i i}\right)$, where $H_{i j}$ and $H_{i i}$ are quasiconvex subgroups of the hyperbolic group $G_{i}$ (see (4.1) and (4.2))), and $H_{i j} \cup H_{i i} \subseteq Q_{i}$. Recalling Lemma 4.1 and (4.5), we see that all the assumptions of Lemma 2.9 are satisfied, whence $H_{j}^{h} \cap H_{i}$ must be cyclic for any $h \in G$.

Thus it remains to prove claim (v). The next lemma will be used for this.

Lemma 4.4. If $N$ is a non-cyclic proper finitely generated subgroup of $G$, then there exists $j \in \mathbb{N} \cup\{0\}$ and $v \in G$ such that $N \subseteq H_{j}^{v}$.

Proof of Lemma 4.4. By construction, there must exist $i \in \mathbb{N}$ such that $N=\psi\left(Y_{i}\right)=\xi_{i}\left(Z_{i}\right)$. Since $N$ is proper and non-cyclic in $G, Z_{i}$ will be a proper non-cyclic subgroup of $G_{i}$. In view of (4.8), the latter implies that $\left|Z_{i}:\left(Z_{i} \cap H_{i j}^{u}\right)\right|<\infty$ for some $j=0,1, \ldots, i$, and some $u \in G_{i}$. Hence $\left|N:\left(N \cap H_{j}^{v}\right)\right|<\infty$ in $G$, where $v=\xi_{i}(u) \in G$, so $H_{j}^{v}$ contains a finite index normal subgroup of $N$. Since $G$ is torsion-free and $H_{j}^{v}$ is malnormal, $N$ must be contained in $H_{j}^{v}$.

To finish the proof of Theorem 1.4, it remains to show that every proper subgroup $M<G$ is contained in a conjugate of some $H_{j}, j \in \mathbb{N} \cup\{0\}$. If $M$ is cyclic, this follows from claim (iii) of the theorem, so we can suppose that $M$ is non-cyclic. Take any $x \in M \backslash\{1\}$. By claim (iii), $x \in H_{k}^{G}$, for some $k \in \mathbb{N} \cup\{0\}$, and, after replacing $M$ with a conjugate, we can assume that $x \in H_{k}$. Then $\mathrm{C}_{G}(x)$ is cyclic by Lemma 4.2 and Lemma 2.4. Since $M$ is non-cyclic, it cannot be contained in $\mathrm{C}_{G}(x)$, so there must exist $y \in M$ such that $N=\langle x, y\rangle \leqslant M$ is non-abelian. Hence $N \subseteq H_{j}^{v}$ for some $j \in \mathbb{N} \cup\{0\}$ and some $v \in G$, by Lemma 4.4.

Now, consider any $z \in M$. The subgroup $P=\langle x, y, z\rangle$ is non-cyclic and proper in $G$, so, according to Lemma 4.4, there exist $i \in \mathbb{N} \cup\{0\}$ and $u \in G$ such that $P \subseteq H_{i}^{u}$. Observe that $N \subseteq H_{j}^{v} \cap H_{i}^{u}$ is non-cyclic, hence $i=j$, by Lemma 4.3 , and $H_{j}^{u}=H_{j}^{v}$, as $H_{j}$ is malnormal in $G$. It follows that $z \in P \subseteq H_{j}^{v}$, whence $M \subseteq H_{j}^{v}$, as required.

Thus we have shown that $G$ satisfies claim (v), so the proof of Theorem 1.4 is complete.

Remark 4.5. It is easy to see that the group $G$ constructed in Theorem 1.4 satisfies the following properties:

- every proper subgroup of $G$ is free;

- $G$ is simple;

- the centralizers of non-trivial elements are cyclic.

Similarly to Remark 3.2, with extra work we can impose additional properties on $G$, such as lacunary hyperbolicity and being a quotient of any given (or, even, every) non-cyclic torsion-free hyperbolic group. 


\section{REFERENCES}

[1] J. Alonso, T. Brady, D. Cooper, V. Ferlini, M. Lustig, M. Mihalik, M. Shapiro, H. Short, Notes on word hyperbolic groups. Edited by Short. Group theory from a geometrical viewpoint (Trieste, 1990), 3-63, World Sci. Publ., River Edge, NJ, 1991.

[2] M. Bestvina, M. Feighn, Addendum and correction to: "A combination theorem for negatively curved groups" [J. Differential Geom. 35 (1992), no. 1, 85-101]. J. Differential Geom. 43 (1996), no. 4, 783-788.

[3] M. Bestvina, K. Fujiwara, Handlebody subgroups in a mapping class group. In the tradition of Ahlfors-Bers. VII, 29-50, Contemp. Math. 696, Amer. Math. Soc., Providence, RI, 2017.

[4] M.R. Bridson, A. Haefliger, Metric spaces of non-positive curvature. Grundlehren der Mathematischen Wissenschaften, 319. Springer-Verlag, Berlin, 1999.

[5] C. Cox, Invariable generation and wreath products. Preprint (2020). arXiv:2001.04748

[6] J.D. Dixon, Random sets which invariably generate the symmetric group. Discrete Math. 105 (1992), no. 1-3, 25-39.

[7] T. Gelander, Convergence groups are not invariably generated. Int. Math. Res. Not. 2015 (2015), no. 19, 9806-9814.

[8] T. Gelander, G. Golan, K. Juschenko, Invariable generation of Thompson groups. J. Algebra 478 (2017), 261-270.

[9] G. Goffer, N. Lazarovich, Invariable generation does not pass to finite index subgroups. Preprint (2020). arXiv:2006.05523

[10] M. Gromov, Hyperbolic groups. Essays in group theory, 75-263, Math. Sci. Res. Inst. Publ., 8, Springer, New York, 1987.

[11] M. Hull, Small cancellation in acylindrically hyperbolic groups. Groups Geom. Dyn. 10 (2016), no. 4, 10771119.

[12] C. Jordan, Recherches sur les substitutions. J. Math. Pures Appl. (2) 17 (1872), 351-367.

[13] W.M. Kantor, A. Lubotzky, A. Shalev, Invariable generation of infinite groups. J. Algebra 421 (2015), 296-310.

[14] O. Kharlampovich, A. Myasnikov, Hyperbolic groups and free constructions. Trans. Amer. Math. Soc. (2) 350 (1998), no. 2, 571-613.

[15] R.C. Lyndon, P.E. Schupp, Paul E. Combinatorial group theory. Ergebnisse der Mathematik und ihrer Grenzgebiete, Band 89. Springer-Verlag, Berlin-New York, 1977.

[16] A. Minasyan, On products of quasiconvex subgroups in hyperbolic groups. Internat. J. Algebra Comput. 14 (2004), no. 2, 173-195.

[17] A. Minasyan, On quasiconvex subsets of hyperbolic groups. Ph.D. Thesis, Vanderbilt University, Nashville, TN, 2005. Available from https://etd.library.vanderbilt.edu.

[18] A. Minasyan, On residualizing homomorphisms preserving quasiconvexity. Comm. Algebra 33 (2005), no. 7, 2423-2463.

[19] A. Minasyan, Some properties of subsets of hyperbolic groups. Comm. Algebra 33 (2005), no. 3, 909-935.

[20] A. Minasyan, P. Zalesskii, One-relator groups with torsion are conjugacy separable. J. Algebra 382 (2013), 39-45.

[21] A.Yu. Ol'shanskii, On residualing homomorphisms and G-subgroups of hyperbolic groups. Internat. J. Algebra and Comput. 3 (1993), no. 4, 365-409.

[22] A.Yu. Olshanskii, D.V. Osin, M.V. Sapir, Lacunary hyperbolic groups. With an appendix by M. Kapovich and B. Kleiner. Geom.E Topol. 13 (2009), no. 4, 2051-2140.

[23] D. Osin, Small cancellations over relatively hyperbolic groups and embedding theorems. Ann. of Math. (2) 172 (2010), no. 1, 1-39.

[24] J.-P. Serre, On a theorem of Jordan. Bull. Amer. Math. Soc. (N.S.) 40 (2003), no. 4, 429-440.

[25] H. Short, Quasiconvexity and a theorem of Howson's. Group theory from a geometrical viewpoint (Trieste, 1990), 168-176, World Sci. Publ., River Edge, NJ, 1991.

[26] J. Wiegold, Transitive groups with fixed-point-free permutations, Arch. Math. (Basel) 27 (1976), no. 5, 473475.

[27] J. Wiegold, Transitive groups with fixed-point-free permutations. II. Arch. Math. (Basel) 29 (1977), no. 6, 571-573.

School of Mathematical Sciences, University of Southampton, Highfield, Southampton, SO17 1BJ, United Kingdom.

E-mail address: aminasyan@gmail.com 